\title{
RIEMANNIAN MANIFOLDS ADMITTING A CONFORMAL TRANSFORMATION GROUP
}

\author{
KENTARO YANO \& SUMIO SAWAKI
}

\section{Introduction}

The purpose of the present paper is to generalize some of the known results on Riemannian manifolds with constant scalar curvature admitting a group of nonisometric conformal transformations.

Let $M$ be a connected Riemannian manifold of dimension $n$, and $g_{j i}, \nabla_{i}$, $K_{k j i}{ }^{h}, K_{j i}=K_{t j i}{ }^{t}$ and $K=K_{j i} g^{j i}$, respectively, the positive definite fundamental metric tensor, the operator of covariant differentiation with respect to the Levi-Civita connection, the curvature tensor, the Ricci tensor and the scalar curvature of $M$, where and in the sequel the indices $h, i, j, k, \cdots$ run over the range $1, \cdots, n$.

If we put

$$
\begin{gathered}
G_{j i}=K_{j i}-\frac{K}{n} g_{j i}, \\
Z_{k j i}^{h}=K_{k j i}^{h}-\frac{K}{n(n-1)}\left(\delta_{k}^{h} g_{j i}-\delta_{j}^{h} g_{k i}\right),
\end{gathered}
$$

we have

$$
Z_{t j i}{ }^{t}=G_{j i}, \quad G_{j i} g^{j i}=0 .
$$

When $M$ admits an infinitesimal transformation $v^{h}$, we denote by $\mathscr{L}$ the operator of Lie derivation with respect to $v^{h}$. Thus, if $M$ admits an infinitesimal conformal transformation $v^{h}$, we have

$$
\mathscr{L} g_{j i}=\nabla_{j} v_{i}+\nabla_{i} v_{j}=2 \rho g_{j i}, \quad \mathscr{L} g^{i h}=-2 \rho g^{i h}
$$

for a certain scalar field $\rho$. We denote the gradient of $\rho$ by $\rho_{i}=\nabla_{i} \rho$.

For an infinitesimal conformal transformation $v^{h}$ in $M$, we have [5]

$$
\begin{gathered}
\mathscr{L} K_{k j i}^{h}=-\delta_{k}^{h} \nabla_{j} \rho_{i}+\delta_{j}^{h} \nabla_{k} \rho_{i}-\nabla_{k} \rho^{h} g_{j i}+\nabla_{j} \rho^{h} g_{k i}, \\
\mathscr{L} K_{j i}=-(n-2) \nabla_{j} \rho_{i}-\Delta \rho g_{j i},
\end{gathered}
$$

Communicated October 19, 1967. 


$$
\mathscr{L} K=-2(n-1) \Delta \rho-2 \rho K
$$

where

$$
\Delta \rho=g^{j i} \nabla_{j} \nabla_{i} \rho
$$

Thus, in $M$ with $K=$ const. we have

$$
\Delta \rho=-\frac{K}{n-1} \rho
$$

We also have

$$
\begin{aligned}
\mathscr{L} G_{j i} & =-(n-2)\left(\nabla_{j} \rho_{i}-\frac{1}{n} \Delta \rho g_{j i}\right), \\
\mathscr{L} Z_{k j i}{ }^{h}= & -\delta_{k}^{h} \nabla_{j} \rho_{i}+\delta_{j}^{h} \nabla_{k} \rho_{i}-\nabla_{k} \rho^{h} g_{j i}+\nabla_{j} \rho^{h} g_{k i} \\
& +\frac{2}{n} \Delta \rho\left(\delta_{k}^{h} g_{j i}-\delta_{j}^{h} g_{k i}\right) .
\end{aligned}
$$

Thus, in $M$ with $K=$ const. we have

$$
\mathscr{L} G_{j i}=-(n-2)\left[\nabla_{j} \rho_{i}+\frac{K}{n(n-1)} \rho g_{j i}\right] .
$$

We denote by $C_{0}(M)$ the largest connected group of conformal transformations of $M$ and by $I_{0}(M)$ that of isometries of $M$.

We first state here known results on Riemannian manifolds with $K=$ const. admitting a conformal transformation group, and then try to generalize them.

Theorem A (Lichnerowicz [3]). If $M$ is a compact Riemannian manifold of dimension $n>2, K=$ const., $K_{j i} K^{j i}=$ const., and $C_{0}(M) \neq I_{0}(M)$, then $M$ is isometric to a sphere.

Theorem B (Lichnerowicz [3], Yano \& Obata [7]). If a compact Riemannian manifold $M$ of dimension $n \geq 2$ with $K=$ const. admits an infinitesimal nonisometric conformal transformation $v^{h}: \mathscr{L}_{j i}=2 \rho g_{j i}, \rho \neq$ const., and if one of the following conditions is satisfied, then $M$ is isometric to a sphere:

(1) The vector field $v^{h}$ is a gradient of a scalar.

(2) $K_{i}{ }^{h} \rho^{i}=k \rho^{h}, k$ being a constant.

(3) $\mathscr{L} K_{j i}=\alpha g_{j i}, \alpha$ being a scalar field.

Theorem $\mathrm{C}$ (Hsiung [1]). If $M$ is compact and of dimension $n>2$, $K=$ const., $K_{k j i h} K^{k j i h}=$ const., and $C_{0}(M) \neq I_{0}(M)$, then $M$ is isometric to a sphere.

Theorem D (Yano [6]). If $M$ is compact orientable and of dimension $n>2$ with $K=$ const., and admits an infinitesimal nonisometric conformal 
transformation $v^{h}: \mathscr{L} g_{j i}=2 \rho g_{j i}, \rho \neq$ const., such that $\int_{M} G_{j i} \rho^{j} \rho^{i} d V$ is nonnegative, $d V$ being the volume element of $M$, then $M$ is isometric to a sphere.

Theorem E (Yano [6]). If $M$ is a compact and of dimension $n>2$ with $K=$ const., and admits an infinitesimal nonisometric conformal transformation $v^{h}: \mathscr{L} g_{j i}=2 \rho g_{j i}, \rho \neq$ const., such that $\mathscr{L}\left(G_{j i} G^{j i}\right)=$ const. or $\mathscr{L}\left(Z_{k j i n} Z^{k_{j i h}}\right)$ $=$ const., then $M$ is isometric to a sphere.

Theorem F (Hsiung [2]). Suppose that a compact Riemannian manifold $M$ of dimension $n>2$ with $K=$ const. admits an infinitesimal nonhomothetic conformal transformation $v^{h}$. If

$$
a^{2} \mathscr{L}\left(Z_{k j i h} Z^{k j i h}\right)+(2 a+n b) b \mathscr{L}\left(G_{j i} G^{j i}\right)=\text { const. }
$$

where $a$ and $b$ are constants such that

$$
c \equiv 4 a^{2}+2(n-2) a b+n(n-2) b^{2}>0,
$$

then $M$ is isometric to a sphere.

To prove and generalize these theorems, we need the following

Theorem G (Obata [4]). If a complete Riemannian manifold of dimension $n \geq 2$ admits a nonconstant function $\rho$ such that

$$
\nabla_{j} \nabla_{i} \rho=-c^{2} \rho g_{j i},
$$

where $c$ is a positive constant, then $M$ is isometric to a sphere of radius $1 / c$ in $(n+1)$-dimensional Euclidean space.

We also need following integral formulas proved in [6].

If a compact orientable Riemannian manifold $M$ of dimension $n>2$ with $K=$ const. admits an infinitesimal nonhomothetic conformal transformation $v^{h}: \mathscr{L} g_{j i}=2 \rho g_{j i}, \rho \neq$ const., then we have

$$
\begin{aligned}
& \int_{M} G_{j i} \rho^{j} \rho^{i} d V=\frac{1}{n-2} \int_{M}\left[2 \rho^{2} G_{j i} G^{j i}+\frac{1}{2} \rho \mathscr{L}\left(G_{j i} G^{j i}\right)\right] d V \\
& \int_{M} G_{j i} \rho^{j} \rho^{i} d V=\int_{M}\left[\frac{1}{2} \rho^{2} Z_{k j i h} Z^{k j i h}+\frac{1}{8} \rho \mathscr{L}\left(Z_{k j i h} Z^{k j i h}\right)\right] d V .
\end{aligned}
$$

\section{Generalization of Theorem B, (2), (3)}

Theorem 2.1. If a compact orientable $M$ of dimension $n>2$ with $K$ $=$ const. admits an infinitesimal nonhomothetic conformal transformation $v^{h}: \mathscr{L}_{j i}=2 \rho g_{j i}, \rho \neq$ const., such that 


$$
\mathscr{L}\left(G^{j i} \mathscr{L} G_{j i}\right) \leq 0,
$$

then $M$ is isometric to a sphere.

We need the following

Lemma 2.1. If a compact orientable $M$ admits an infinitesimal conformal tsansformation $v^{h}: \mathscr{L}_{j i}=2 \rho g_{j i}$, then we have

$$
\int_{M} \rho F d V=-\frac{1}{n} \int_{M} \mathscr{L} F d V
$$

for any function $F$.

Proof. Since $\rho=\frac{1}{n} \nabla_{s} v^{s}$, we have, by Green's theorem,

$$
\begin{aligned}
\int_{M} \rho F d V & =\frac{1}{n} \int_{M}\left(\nabla_{s} v^{s}\right) F d V \\
& =-\frac{1}{n} \int_{M} v^{s} \nabla_{s} F d V \\
& =-\frac{1}{n} \int_{M} \mathscr{L} F d V .
\end{aligned}
$$

Proof of the Theorem. Substituting

$$
\mathscr{L}\left(G_{j i} G^{j i}\right)=2 G^{j i} \mathscr{L} G_{j i}-4 \rho G^{j i} G_{j i}
$$

into integral formula (1.16), we find

$$
\int_{M} G_{j i} \rho^{j} \rho^{i} d V=\frac{1}{n-2} \int_{M} \rho G^{j i} \mathscr{L} G_{j i} d V .
$$

Consequently, by Lemma 2.1 and the assumption of the theorem, we have

$$
\int_{M} G_{j i} \rho^{j} \rho^{i} d V=-\frac{1}{n(n-2)} \int_{M} \mathscr{L}\left(G^{j i} \mathscr{L} G_{j i}\right) d V \geq 0 .
$$

Thus $M$ is isometric to a sphere by Theorem $\mathrm{D}$.

Remark 2.1. Since

$$
Z^{k j i}{ }_{h} \mathscr{L} Z_{k j i}{ }^{h}=\frac{4}{n-2} G^{j i} \mathscr{L} G_{j i},
$$

the condition (2.1) of the theorem can be replaced by

$$
\mathscr{L}\left(Z^{k j i}{ }_{h} \mathscr{L} Z_{k j i}{ }^{h}\right) \leq 0 \text {. }
$$


Remark 2.2. As the proof of the theorem shows, condition (2.1) can be replaced by

$$
\mathscr{L}\left(G^{j i} \mathscr{L} G_{j i}\right)=\lambda, \quad \int_{M} \lambda d V \leq 0
$$

The same remark applies to Theorems 4.1, 4.3, 5.1, 6.1, 6.2 and 6.4.

Remark 2.3. Theorem 2.1 generalizes Theorem B, (2). In fact, using $K_{i}^{h} \rho^{i}=k \rho^{h}, \nabla_{j} K^{j i}=0, \nabla_{j} v_{i}+\nabla_{i} v_{j}=2 \rho g_{j i}$ and $\nabla_{i} v^{i}=n \rho$, we have

$$
\begin{aligned}
\nabla_{j}\left(K^{j i} \rho v_{i}\right) & =K^{j i} \rho_{j} v_{i}+K^{j i} \rho \nabla_{j} v_{i} \\
& =k \rho_{i} v^{i}+\frac{1}{2} K^{j i} \rho\left(\nabla_{j} v_{i}+\nabla_{i} v_{j}\right) \\
& =k \nabla_{i}\left(\rho v^{i}\right)-k \rho \nabla_{i} v^{i}+K \rho^{2} \\
& =k \nabla_{i}\left(\rho v^{i}\right)-n k \rho^{2}+K \rho^{2},
\end{aligned}
$$

from which, by integration,

$$
\int_{M}(K-n k) \rho^{2} d V=0,
$$

and consequently $k=K / n$.

Thus, from $K_{i}{ }^{h} \rho^{i}=k \rho^{h}$ we have

$$
\begin{aligned}
& \left(K^{j i}-\frac{K}{n} g^{j i}\right) \rho_{i}=0 \\
& \left(K^{j i}-\frac{K}{n} g^{j i}\right) \nabla_{j} \rho_{i}=0
\end{aligned}
$$

and consequently, by virtue of (1.10),

$$
G^{j i}\left(\mathscr{L} G_{j i}\right)=0 .
$$

Remark 2.4. Theorem 2.1 generalizes Theorem B, (3). In fact, from (1.6) and $\mathscr{L} K_{j i}=\alpha g_{j i}$ we find

$$
-(n-2) \nabla_{j} \rho_{i}-\Delta \rho g_{j i}=\alpha g_{j i},
$$

from which

$$
\alpha=-2(n-1) \Delta \rho / n,
$$

and consequently

$$
-(n-2)\left(\nabla_{j} \rho_{i}-\frac{1}{n} \Delta \rho g_{j i}\right)=0,
$$

that is, $\mathscr{L} G_{j i}=0$. 
Remark 2.5. If $G^{j i} \mathscr{L} G_{j i}=$ const., then (2.1) is automatically satisfied, but under our assumption the constant must be zero. In fact, making use of (1.3) and $\nabla_{j} G^{j i}=0$, from (1.10) we have

$$
\begin{aligned}
G^{j i} \mathscr{L} G_{j i} & =-(n-2) G^{i j} \nabla_{j} \rho_{i} \\
& =-(n-2) \nabla_{j}\left(G^{j i} \rho_{i}\right),
\end{aligned}
$$

and consequently by integration over $M$ we find

$$
\int_{M} G^{j i} \mathscr{L} G_{j i} d V=0 .
$$

Thus, if $G^{j i} \mathscr{L} G_{j i}=$ const. the constant must be zero.

\section{Decomposition of a conformal Killing vector}

Theorem 3.1. If a compact orientable $M$ of dimension $n \geq 2$ with $K$ $=$ const. admits a conformal Killing vector field

$$
v^{h}=p^{h}+q^{h},
$$

where $p^{h}$ is a Killing vector field and $q^{h}=\nabla^{h} q, q \neq$ const. is a gradient conformal Killing vector field, then $M$ is isometric to a sphere.

Conversely, if a sphere of dimension $n \geq 2$ admits a conformal Killing vector field $v^{h}$, then $v^{h}$ is decomposed into the form (3.1) where $p^{h}$ is a Killing vector field and $q^{h}$ a gradient conformal Killing vector field.

Proof. Suppose that a compact orientable $M$ with $K=$ const. admits a conformal Killing vector $v^{h}$. Then we have

$$
\mathscr{L} g_{j i}=\nabla_{j} v_{i}+\nabla_{i} v_{j}=2 \rho g_{j i},
$$

and

$$
\Delta \rho=-\frac{K}{n-1} \rho
$$

We note here that $K$ is a positive constant [6]. If $v^{h}$ is the sum of a Killing vector $p^{h}$ and a gradient conformal Killing vector $q^{h}=\nabla^{h} q$, substituting (3.1) into (3.2), we find

$$
\nabla_{j} \nabla_{i} q=\rho g_{j i},
$$

from which

$$
\Delta q=n \rho .
$$

From (3.3) and (3.5), we find 


$$
\Delta\left(\rho+\frac{K}{n(n-1)} q\right)=0
$$

from which, by Bochner's lemma,

$$
\rho+\frac{K}{n(n-1)} q=\text { constant } .
$$

Substituting (3.6) into (3.4), we find

$$
\nabla_{j} \nabla_{i}(q+c)=-\frac{K}{n(n-1)}(q+c) g_{j i}
$$

where $c$ is a constant. Thus, $q$ being not a constant, $M$ is isometric to a sphere.

Conversely, suppose that $M$, isometric to a sphere, admits a conformal Killing vector $v^{h}$. It is known that $v^{h}$ can be decomposed into

$$
v^{h}=p^{h}+q^{h}
$$

where

$$
\nabla_{i} p^{i}=0, \quad q^{h}=\nabla^{h} q
$$

From

$$
\mathscr{L} g_{i i}=\nabla_{i} v_{i}+\nabla_{i} v_{j}=2 \rho g_{j i},
$$

we have

$$
T_{j i}=\nabla_{j} p_{i}+\nabla_{i} p_{j}+2 \nabla_{j} \nabla_{i} q-2 \rho g_{j i}=0 .
$$

Forming $T_{j i} T^{j i}$, we find

$$
\begin{aligned}
T_{j i} T^{j i}= & \left(\nabla_{j} p_{i}+\nabla_{i} p_{j}\right)\left(\nabla^{j} p^{i}+\nabla^{i} p^{j}\right) \\
& +4\left(\nabla_{j} \nabla_{i} q-\frac{1}{n} \Delta q g_{j i}\right)\left(\nabla^{i} \nabla^{i} q-\frac{1}{n} \Delta q g^{j i}\right) \\
& +8\left(\nabla^{j} \nabla^{i} q\right)\left(\nabla_{j} p_{i}\right)=0 .
\end{aligned}
$$

On the other hand, we have

$$
\begin{aligned}
\int_{M}\left(\nabla^{j} \nabla^{i} q\right)\left(\nabla_{j} p_{i}\right) d V & =\int_{M}\left(\nabla^{i} \nabla^{j} q\right)\left(\nabla_{j} p_{i}\right) d V \\
& =-\int_{M}\left(\nabla^{j} q\right)\left(\nabla^{i} \nabla_{j} p_{i}\right) d V \\
& =-\int_{M} K_{j i}\left(\nabla^{j} q\right) p^{i} d V
\end{aligned}
$$

because of 


$$
\nabla_{i} \nabla_{j} p^{i}-\nabla_{j} \nabla_{i} p^{i}=K_{i j t}{ }^{i} p^{\ell}
$$

or

$$
\nabla^{i} \nabla_{j} p_{i}=K_{j t} p^{t}
$$

Taking account of $K_{j i}=\frac{K}{n} g_{j i}$ we then have

$$
\begin{aligned}
\int_{M}\left(\nabla^{j} \nabla^{i} q\right)\left(\nabla_{j} p_{i}\right) d V & =-\frac{K}{n} \int_{M}\left(\nabla_{i} q\right) p^{i} d V \\
& =\frac{K}{n} \int_{M} q\left(\nabla_{i} p^{i}\right) d V \\
& =0 .
\end{aligned}
$$

Thus from (3.9), by integration we find

$$
\begin{aligned}
& \int_{M}\left[\left(\nabla_{j} p_{i}+\nabla_{i} p_{j}\right)\left(\nabla^{j} p^{i}+\nabla^{i} p^{j}\right)\right. \\
& \left.\quad+4\left(\nabla_{j} \nabla_{i} q-\frac{1}{n} \Delta q g_{j i}\right)\left(\nabla^{j} \nabla^{i} q-\frac{1}{n} \Delta q g^{j i}\right)\right] d V=0,
\end{aligned}
$$

from which

$$
\begin{aligned}
& \nabla_{j} p_{i}+\nabla_{i} p_{j}=0, \\
& \nabla_{j} \nabla_{i} q=\frac{1}{n} \Delta q g_{j i},
\end{aligned}
$$

showing that $p^{h}$ is a Killing vector field and $q^{h}$ a gradient conformal Killing vector field.

Remark 3.1. Theorem 3.1 generalizes Theorem B, (1).

Remark 3.2. We can see in the following way the fact that a sphere admits a gradient conformal Killing vector field. Let

$$
X^{A}=X^{A}\left(x^{h}\right), \quad \sum X^{A} X^{A}=r^{2}
$$

be the equations of $n$-dimensional sphere of radius $r$ in an $(n+1)$-dimensional Euclidean space, where $A=1, \cdots, n+1$.

The equations of Gauss and those of Weingarten of the shpere are, respectively,

$$
\nabla_{j} B_{i}{ }^{\Lambda}=\frac{1}{r} g_{j i} N^{\Lambda}
$$

and 


$$
\nabla_{j} N^{A}=-\frac{1}{r} B_{j}{ }^{A},
$$

where $B_{i}{ }^{A}=\nabla_{i} X^{A}$ and $N^{A}$ are components of the unit normal to the sphere.

Considering a parallel vector field $B_{i}{ }^{A} u^{i}+\alpha N^{A}$ in the Euclidean space along the sphere, we have

$$
\nabla_{j}\left(B_{i}{ }^{A} u^{i}+\alpha N^{A}\right)=0
$$

from which

$$
\frac{1}{r} u_{j} N^{A}+B_{i}{ }^{A} \nabla_{j} u^{i}+\left(\nabla_{j} \alpha\right) N^{A}-\frac{\alpha}{r} B_{j}{ }^{A}=0,
$$

and consequently

$$
\nabla_{j} u^{i}=\frac{\alpha}{r} \delta_{j}^{i}, \quad \Delta_{j} \alpha=-\frac{1}{r} u_{j},
$$

thus giving

$$
\nabla_{j} \nabla_{i} \alpha=-\frac{1}{r} \nabla_{j} u_{i}
$$

that is,

$$
\nabla_{j} \nabla_{i} \alpha=-\frac{1}{r^{2}} \alpha g_{j i}
$$

\section{Generalizations of Theorem $\mathbf{E}$}

We introduce here the notations:

$$
f=G_{j i} G^{j i}, \quad g=Z_{k j i h} Z^{k j i h} .
$$

Theorem 4.1. If a compact orientable $M$ of dimension $n>2$ with $K=$ const. admits an infinitesimal nonhomothetic conformal transformation $v^{h}$ such that

$$
\begin{aligned}
\mathscr{L}\left\{\sum_{k=0}^{l} \alpha_{k}\left(-\frac{n-1}{K}\right)^{k} \Delta^{k}(\mathscr{L} f)\right. \\
\left.+\sum_{k=0}^{m} \beta_{k}\left(-\frac{n-1}{K}\right)^{k} \Delta^{k}(\mathscr{L} g)\right\} \leq 0,
\end{aligned}
$$

$l$ and $m$ being nonnegative integers, and $\alpha_{k}$ and $\beta_{k}$ constants such that the sums $\sum_{k=0}^{l} \alpha_{k}$ and $\sum_{k=0}^{m} \beta_{k}$ are nonnegative and not both zero, then $M$ is isometric to a sphere. 
We need the following

Lemma 4.1. If a compact orientable $M$ with $K=$ const. admits an infinitesimal conformal transformation $v^{h}: \mathscr{L}_{j i}=2 \rho g_{i i}$, then we have

$$
\begin{aligned}
\int_{M} \rho F d V= & \int_{M}\left(-\frac{n-1}{K}\right) \rho \Delta F d V \\
= & \int_{M}\left(-\frac{n-1}{K}\right)^{2} \rho \Delta^{2} F d V \\
& \cdots \cdots \\
= & \int_{M}\left(-\frac{n-1}{K}\right)^{l} \rho \Delta^{l} F d V
\end{aligned}
$$

for any function $F$ and any nonnegative integer $l$.

Proof. Remembering

$$
\Delta \rho=-\frac{K}{n-1} \rho \quad(K>0)
$$

that is,

$$
\rho=-\frac{n-1}{K} \Delta \rho,
$$

we have, for any scalar field $F$,

$$
\int_{M} \rho F d V=\int_{M}\left(-\frac{n-1}{K}\right)(\Delta \rho) F d V,
$$

that is,

$$
\int_{M} \rho F d V=\int_{M}\left(-\frac{n-1}{K}\right) \rho \Delta F d V \text {. }
$$

Repeating the same process, we hence obtain (4.3).

Proof of the theorem. We have, from (1.16) and Lemma 4.1,

$$
\begin{aligned}
\frac{n-2}{2} \int_{M} G_{j i} \rho^{j} \rho^{i} d V & =\int_{M} \rho^{2} f d V+\frac{1}{4} \int_{M} \rho \mathscr{L} f d V \\
& =\int_{M} \rho^{2} f d V+\frac{1}{4} \int_{M}\left(-\frac{n-1}{K}\right) \rho \Delta \mathscr{L} f d V \\
& \ldots \cdots \cdots \\
& =\int_{M} \rho^{2} f d V+\frac{1}{4} \int_{M}\left(-\frac{n-1}{K}\right)^{l} \rho \Delta^{l} \mathscr{L} f d V .
\end{aligned}
$$


We also have, from (1.17) and Lemma 4.1,

$$
\begin{aligned}
2 \int_{M} G_{j i} \rho^{j} \rho^{i} d V & =\int_{M} \rho^{2} g d V+\frac{1}{4} \int_{M} \rho \mathscr{L} g d V \\
& =\int_{M} \rho^{2} g d V+\frac{1}{4} \int_{M}\left(-\frac{n-1}{K}\right) \rho \Delta \mathscr{L} g d V \\
& \ldots \ldots \ldots \\
& =\int_{M} \rho^{2} g d V+\frac{1}{4} \int_{M}\left(-\frac{n-1}{K}\right)^{m} \rho \Delta^{m} \mathscr{L} g d V .
\end{aligned}
$$

From these equations, we have

$$
\begin{aligned}
& \left\{\frac{n-2}{2}\left(\alpha_{0}+\alpha_{1}+\cdots+\alpha_{l}\right)+2\left(\beta_{0}+\beta_{1}+\cdots+\beta_{m}\right)\right\} \int_{M} G_{j i} \rho^{j} \rho^{i} d V \\
& =\int_{\mathscr{M}} \rho^{2}\left\{\left(\alpha_{0}+\alpha_{1}+\cdots+\alpha_{l}\right) f+\left(\beta_{0}+\beta_{1}+\cdots+\beta_{m}\right) g\right\} d V \\
& \quad+\frac{1}{4} \int_{M} \rho\left\{\alpha_{0} \mathscr{L} f+\alpha_{1}\left(-\frac{n-1}{K}\right) \Delta \mathscr{L} f+\cdots+\alpha_{l}\left(-\frac{n-1}{K}\right)^{l} \Delta^{l} \mathscr{L} f\right. \\
& +\beta_{0} \mathscr{L} g+\beta_{1}\left(-\frac{n-1}{K}\right) \Delta \mathscr{L} g+\cdots \\
& \left.+\beta_{m}\left(-\frac{n-1}{K}\right)^{m} \Delta^{m} \mathscr{L} g\right\} d V,
\end{aligned}
$$

and consequently, by Lemma 2.1 ,

$$
\begin{gathered}
\left\{\frac{n-2}{2}\left(\alpha_{0}+\alpha_{1}+\cdots+\alpha_{l}\right)+2\left(\beta_{0}+\beta_{1}+\cdots \beta_{m}\right)\right\} \int_{M} G_{j i} \rho^{j} \rho^{i} d V \\
=\int_{M} \rho^{2}\left\{\left(\alpha_{0}+\alpha_{1}+\cdots+\alpha_{l}\right) f+\left(\beta_{0}+\beta_{1}+\cdots+\beta_{m}\right) g\right\} d V \\
\quad-\frac{1}{4 n} \int_{M} \mathscr{L}\left\{\alpha_{0} \mathscr{L} f+\alpha_{1}\left(-\frac{n-1}{K}\right) \Delta \mathscr{L} f+\cdots\right. \\
+\alpha_{l}\left(-\frac{n-1}{K}\right)^{l} \Delta^{l} \mathscr{L} f+\beta_{0} \mathscr{L} g \\
+\beta_{1}\left(-\frac{n-1}{K}\right)^{\Delta \mathscr{L} g}+\cdots \\
\left.+\beta_{m}\left(-\frac{n-1}{K}\right)^{m} \Delta^{m} \mathscr{L} g\right\} d V .
\end{gathered}
$$

Thus, if the conditions of the theorem are satisfied, we have 


$$
\int_{M} G_{j i} \rho^{j} \rho^{i} d V \geq 0
$$

and consequently, by Theorem $\mathrm{D}, M$ is isometric to a sphere.

Theorem 4.2. Suppose that a compact orientable $M$ of dimension $n>2$ with $K=$ const. satisfies

$$
\alpha_{0} f-\alpha_{1} \Delta f+\beta_{0} g-\beta_{1} \Delta g=\text { const. },
$$

where $\alpha_{0}, \alpha_{1}, \beta_{0}, \beta_{1}$ are nonnegative constants not all zero such that, if $n>6$,

$$
\frac{8 K}{n-1} \alpha_{1} \geq(n-6) \alpha_{0} \geq 0, \quad \frac{8 K}{n-1} \beta_{1} \geq(n-6) \beta_{0} \geq 0 \text {. }
$$

If $M$ admits an infinitesimal nonhomothetic conformal transformation $v^{h}$ : $\mathscr{L} g_{i i}=2 \rho g_{j i}, \rho \neq$ constant, then $M$ is isometric to a sphere.

To prove the theorem, we need the following

Lemma 4.2. For a conformal Killing vector $v^{h}$ in $M$, that is, for a vector field $v^{h}$ satisfying

$$
\mathscr{L}_{j i}=\nabla_{j} v_{i}+\nabla_{i} v_{j}=2 \rho g_{j i},
$$

we heve

$$
\Delta(\mathscr{L} F)=\mathscr{L}(\Delta F)+2 \rho \Delta F-(n-2) \rho^{i} \nabla_{i} F
$$

for any scalar field $F$.

Proof. Since $v^{h}$ is a conformal Killing vector field, we have

$$
g^{j i} \nabla_{j} \nabla_{i} v^{h}+K_{i}^{h} v^{i}+(n-2) \rho^{h}=0,
$$

(see [6] for example). We also have, for an arbitrary scalar field $F$,

$$
g^{j i} \nabla_{j} \nabla_{i} \nabla_{h} F-K_{h}^{i} \nabla_{i} F=\nabla_{h}(\Delta F) .
$$

Thus we have

$$
\begin{aligned}
\Delta(\mathscr{L} F)= & g^{j i} \nabla_{j} \nabla_{i}\left(v^{h} \nabla_{h} F\right) \\
= & \left(g^{j i} \nabla_{j} \nabla_{i} v^{h}\right) \nabla_{h} F+\left(\nabla^{j} v^{i}+\nabla^{i} v^{j}\right) \nabla_{j} \nabla_{i} F \\
& +v^{h} g^{j i} \nabla_{j} \nabla_{i} \nabla_{h} F,
\end{aligned}
$$

and consequently, by using (4.7) and (4.8),

$$
\begin{aligned}
\Delta(\mathscr{L} F)= & -K_{j i} v^{i} \nabla^{j} F-(n-2) \rho^{h} \nabla_{h} F \\
& +2 \rho \Delta F+K_{j i} v^{j} \nabla^{i} F+v^{h} \nabla_{h}(\Delta F),
\end{aligned}
$$

that is, 


$$
\Delta(\mathscr{L} F)=\mathscr{L} \Delta F+2 \rho \Delta F-(n-2) \rho^{h} \nabla_{h} F
$$

Lemma 4.3. For any scalar field $F$ and a scalar field $\rho$ satisfying $\Delta \rho=k \rho$, $k$ being a constant, in a compact orientable $M$ we have

$$
\begin{gathered}
\int_{M} \rho \rho^{h} \nabla_{h} F d V=-\frac{1}{2} \int_{M} \rho^{2}(\Delta F) d V, \\
\int_{M} \rho^{2}(\Delta F) d V=2 k \int_{M} \rho^{2} F d V+2 \int_{M} \rho_{i} \rho^{i} F d V .
\end{gathered}
$$

Proof. Integral formula (4.9) follows from

$$
\nabla^{h}\left(\rho^{2} \nabla_{h} F\right)=2 \rho \rho^{h} \nabla_{h} F+\rho^{2} \Delta F
$$

by integration. On the other hand, we have

$$
\begin{aligned}
\int_{M} \rho^{2}(\Delta F) d F & =\int_{M}\left(\Delta \rho^{2}\right) F d V \\
& =2 \int_{M}\left(\rho \Delta \rho+\rho_{i} \rho^{i}\right) F d V \\
& =2 k \int_{M} \rho^{2} F d V+2 \int_{M} \rho_{i} \rho^{i} F d V,
\end{aligned}
$$

which proves (4.10).

Proof of the theorem. From (1.16), (4.3), (4.6) and (4.9), we find

$$
\begin{aligned}
\frac{n-2}{2} \int_{M} G_{j i} \rho^{j} \rho^{i} d V \\
=\int_{M} \rho^{2} f d V+\frac{1}{4} \int_{M} \rho \mathscr{L} f d V \\
=\int_{M} \rho^{2} f d V+\frac{1}{4} \int_{M}\left(-\frac{n-1}{K}\right) \rho \Delta \mathscr{L} f d V \\
=\int_{M} \rho^{2} f d V+\frac{1}{4} \int_{M}\left(-\frac{n-1}{K}\right) \rho\left[\mathscr{L} \Delta f+2 \rho \Delta f-(n-2) \rho^{i} \nabla_{i} f\right] d V \\
=\int_{M} \rho^{2} f d V+\frac{1}{4} \int_{M} \rho \mathscr{L}\left(-\frac{n-1}{K} \Delta f\right) d V \\
+\frac{1}{4} \int_{M}\left[-\frac{2(n-1)}{K} \rho^{2} \Delta f-\frac{(n-1)(n-2)}{2 K} \rho^{2} \Delta f\right] d V \\
=\int_{M} \rho^{2} f d V+\frac{1}{4} \int_{M} \rho \mathscr{L}\left(-\frac{n-1}{K} \Delta f\right) d V-\frac{(n-1)(n+2)}{8 K} \int_{M} \rho^{2} \Delta f d V .
\end{aligned}
$$

Thus, we have 


$$
\begin{aligned}
\frac{n-2}{2} \int_{M} G_{j i} \rho^{j} \rho^{i} d V= & \int_{M} \rho^{2} f d V+\frac{1}{4} \int_{M} \rho \mathscr{L} f d V \\
\frac{n-2}{2}-\int_{M} G_{j i} \rho^{j} \rho^{i} d V= & \int_{M} \rho^{2} f d V+\frac{1}{4} \int_{M} \rho \mathscr{L}\left(-\frac{n-1}{K} \Delta f\right) d V \\
& -\frac{(n-1)(n+2)}{8 K}-\int_{M} \rho^{2} \Delta f d V .
\end{aligned}
$$

Similarly, we find

$$
\begin{aligned}
2 \int_{M} G_{j i} \rho^{j} \rho^{i} d V= & \int_{M} \rho^{2} g d V+\frac{1}{4} \int_{M} \rho \mathscr{L} g d V, \\
2 \int_{M} G_{j i} \rho^{j} \rho^{i} d V= & \int_{M} \rho^{2} g d V+\frac{1}{4} \int_{M} \rho \mathscr{L}\left(-\frac{n-1}{K} \Delta g\right) d V \\
& -\frac{(n-1)(n+2)}{8 K} \int_{M} \rho^{2} \Delta g d V .
\end{aligned}
$$

From the above four equations, we obtain

$$
\begin{aligned}
& \left\{\frac{n-2}{2}\left(a+a^{\prime}\right)+2\left(b+b^{\prime}\right)\right\} \int_{M} G_{j i} \rho^{j} \rho^{i} d V \\
& =\int_{M} \rho^{2}\left[\left(a+a^{\prime}\right) f+\left(b+b^{\prime}\right) g\right] d V \\
& \quad+\frac{1}{4} \int_{M} \rho\left[\mathscr{L}\left(a f-\frac{n-1}{K} a^{\prime} \Delta f+b g-\frac{n-1}{K} b^{\prime} \Delta g\right)\right] d V \\
& \quad-\frac{(n-1)(n+2)}{8 K} \int_{M} \rho^{2}\left(a^{\prime} \Delta f+b^{\prime} \Delta g\right) d V,
\end{aligned}
$$

$a, a^{\prime}, b, b^{\prime}$ being nonnegative constants. Now we choose $a, a^{\prime}, b, b^{\prime}$ in such a way that we have

$$
\alpha_{0}=a, \quad \alpha_{1}=\frac{n-1}{K} a^{\prime}, \quad \beta_{0}=b, \quad \beta_{1}=\frac{n-1}{K} b^{\prime} .
$$

Then we have, from (4.4),

$$
a f-\frac{n-1}{K} a^{\prime} \Delta f+b g-\frac{n-1}{K} b^{\prime} \Delta g=c \text { (const.) }
$$

and 


$$
a^{\prime} \Delta f+b^{\prime} \Delta g=\frac{K}{n-1}(a f+b g)-\frac{K c}{n-1},
$$

and consequently, from (4.11),

$$
\begin{aligned}
\left\{\frac{n-2}{2}\left(a+a^{\prime}\right)+2\left(b+b^{\prime}\right)\right\} \int_{M} G_{j i} \rho^{j} \rho^{i} d V \\
=\int_{M} \rho^{2}\left[\left(a+a^{\prime}\right) f+\left(b+b^{\prime}\right) g\right. \\
\left.\quad-\frac{(n-1)(n+2)}{8 K}\left\{\frac{K}{n-1}(a f+b g)-\frac{K c}{n-1}\right\}\right] d V,
\end{aligned}
$$

that is,

$$
\begin{aligned}
& \left\{\frac{n-2}{2}\left(a+a^{\prime}\right)+2\left(b+b^{\prime}\right)\right\} \int_{M} G_{j i} \rho^{j} \rho^{i} d V \\
& \quad=\frac{1}{8} \int_{M} \rho^{2}\left[\left\{8 a^{\prime}-(n-6) a\right\} f+\left\{8 b^{\prime}-(n-6) b\right\} g+(n+2) c\right] d V .
\end{aligned}
$$

Now, constants

$$
8 a^{\prime}-(n-6) a, \quad 8 b^{\prime}-(n-6) b
$$

are both nonnegative for $n \leq 6$. Since

$$
\begin{aligned}
& 8 a^{\prime}-(n-6) a=\frac{8 K}{n-1} \alpha_{1}-(n-6) \alpha_{0}, \\
& 8 b^{\prime}-(n-6) b=\frac{8 K}{n-1} \beta_{1}-(n-6) \beta_{0},
\end{aligned}
$$

they are nonnegative also for $n \geq 6$ by virtue of the assumption.

Moreover, we have, from (4.13),

$$
a \int_{M} f d V+b \int_{M} g d V=c \int d V,
$$

and consequently $c$ is nonnegative.

Thus we have, from (4.14),

$$
\int_{M} G_{j i} \rho^{j} \rho^{i} d V \geq 0,
$$

and consequently, by Theorem $\mathrm{D}, M$ is isometric to a sphere. 
Theorem 4.3. If a compact orientable $M$ of dimension $n>2$ with $K$ $=$ const. admits an infinitesimal nonhomothetic conformal transformation $v^{h}$ such that

$$
\mathscr{L} \mathscr{L}\left(\alpha_{0} f+\alpha_{1} \Delta f+\beta_{0} g+\beta_{1} \Delta g\right) \leq 0,
$$

$\alpha_{0}, \alpha_{1}, \beta_{0}, \beta_{1}$ being constants not all zero such that

$$
\frac{4(n-1)}{K} \alpha_{0} \geq(n+6) \alpha_{1} \geq 0, \quad \frac{4(n-1)}{K} \beta_{0} \geq(n+6) \beta_{1} \geq 0,
$$

then $M$ is isometric to a sphere.

To prove this theorem, we need the following

Lemma 4.4. If a compact orientable $M$ of dimension $n>2$ with $K$ $=$ const. admits an infinitesimal nonhomothetic conformal transformation $v^{h}$, then

$$
\begin{aligned}
\frac{n-2}{2} \int_{M} G_{j i} \rho^{j} \rho^{i} d V= & \frac{n+6}{4} \int_{M} \rho^{2} f d V-\frac{n-1}{4 K} \int_{M} \rho \mathscr{L} \Delta f d V \\
& -\frac{(n-1)(n+2)}{4 K} \int_{M} \rho_{i} \rho^{i} f d V \\
2 \int_{M} G_{j i} \rho^{j} \rho^{i} d V= & \frac{n+6}{4} \int_{M} \rho^{2} g d V-\frac{n-1}{4 K} \int_{M} \rho \mathscr{L} \Delta g d V \\
& -\frac{(n-1)(n+2)}{4 K} \int_{M} \rho_{i} \rho^{i} g d V .
\end{aligned}
$$

Proof. From (1.16), we have

$$
\frac{n-2}{2} \int_{M} G_{j i} \rho^{j} \rho^{i} d V=\int_{M} \rho^{2} f d V+\frac{1}{4} \int_{M} \rho \mathscr{L} f d V .
$$

Substituting $\rho=-\frac{n-1}{K} \Delta \rho$ into the last term of the second member of this equation, we find

$$
\begin{aligned}
\frac{n-2}{2} \int_{M} G_{j i} \rho^{j} \rho^{i} d V & =\int_{M} \rho^{2} f d V-\frac{n-1}{4 K} \int_{M}(\Delta \rho) \mathscr{L} f d V \\
& =\int_{M} \rho^{2} f d V-\frac{n-1}{4 K} \int_{M} \rho \Delta(\mathscr{L} f) d V,
\end{aligned}
$$

and consequently, by (4.6), 
MANIFOLDS ADMITTING A TRANSFORMATION GROUP

177

$$
\begin{aligned}
\frac{n-2}{2} \int_{M} G_{j i} \rho^{j} \rho^{i} d V= & \int_{M} \rho^{2} f d V \\
& -\frac{n-1}{4 K} \int_{M} \rho\left\{\mathscr{L} \Delta f+2 \rho \Delta f-(n-2) \rho^{i} \nabla_{i} f\right\} d V .
\end{aligned}
$$

Thus by (4.9) and (4.10) with $k=-\frac{K}{n-1}$, we find

$$
\begin{aligned}
\frac{n-2}{2} \int_{M} G_{j i} \rho^{j} \rho^{i} d V= & \int_{M} \rho^{2} f d V-\frac{n-1}{4 K} \int_{M}\left(\rho \mathscr{L} \Delta f+\frac{n+2}{2} \rho^{2} \Delta f\right) d V \\
= & \int_{M} \rho^{2} f d V-\frac{n-1}{4 K} \int_{M} \rho \mathscr{L} \Delta f d V \\
& -\frac{(n-1)(n+2)}{8 K} \int_{M}\left(-\frac{2 K}{n-1} \rho^{2} f+2 \rho_{i} \rho^{i f f}\right) d V \\
= & \frac{n+6}{4} \int_{M} \rho^{2} f d V-\frac{n-1}{4 K} \int_{M} \rho \mathscr{L} \Delta f d V \\
& -\frac{(n-1)(n+2)}{4 K} \int_{M} \rho_{i} \rho^{i} f d V .
\end{aligned}
$$

We can similarly prove (4.18).

Proof of the theorem. We first write down (1.16), (4.17), (1.17) and (4.18):

$$
\begin{aligned}
\frac{n-2}{2} \int_{M} G_{j i} \rho^{j} \rho^{i} d V= & \int_{M} \rho^{2} f d V+\frac{1}{4} \int_{M} \rho \mathscr{L} f d V, \\
\frac{n-2}{2} \int_{M} G_{j i} \rho^{j} \rho^{i} d V= & \frac{n+6}{4} \int_{M} \rho^{2} f d V-\frac{n-1}{4 K} \int_{M} \rho \mathscr{L} \Delta f d V \\
& -\frac{(n-1)(n+2)}{4 K} \int_{M} \rho_{i} \rho^{i} f d V, \\
2 \int_{M} G_{j i} \rho^{j} \rho^{i} d V= & \int_{M} \rho^{2} g d V+\frac{1}{4} \int_{M} \rho \mathscr{L} \mathrm{g} d V, \\
2 \int_{M} G_{j i} \rho^{j} \rho^{i} d V= & \frac{n+6}{4} \int_{M} \rho^{2} g d V-\frac{n-1}{4 K} \int_{M} \rho \mathscr{L} \Delta g d V \\
& -\frac{(n-1)(n+2)}{4 K} \int_{M} \rho_{i} \rho^{i} g d V,
\end{aligned}
$$

from which we obtain 


$$
\begin{aligned}
& \left\{\frac{n-2}{2}\left(a-a^{\prime}\right)+2\left(b-b^{\prime}\right)\right\} \int_{M} G_{j i} \rho^{j} \rho^{i} d V \\
& =\frac{1}{4}\left\{4 a-(n+6) a^{\prime}\right\} \int_{M} \rho^{2} f d V+\frac{1}{4}\left\{4 b-(n+6) b^{\prime}\right\} \int_{M} \rho^{2} g d V \\
& \quad+\frac{1}{4} \int_{M} \rho \mathscr{L}\left(a f+\frac{n-1}{K} a^{\prime} \Delta f+b g+\frac{n-1}{K} b^{\prime} \Delta g\right) d V \\
& \quad+\frac{(n-1)(n+2)}{4 K} \int_{M} \rho_{i} \rho^{i}\left(a^{\prime} f+b^{\prime} g\right) d V,
\end{aligned}
$$

or by Lemma 2.1 ,

$$
\begin{aligned}
& \left\{\frac{n-2}{2}\left(a-a^{\prime}\right)+2\left(b-b^{\prime}\right)\right\} \int_{M} G_{j i} \rho^{j} \rho^{i} d V \\
& =\frac{1}{4}\left\{4 a-(n+6) a^{\prime}\right\} \int_{M} \rho^{2} f d V+\frac{1}{4}\left\{4 b-(n+6) b^{\prime}\right\} \int_{M} \rho^{2} g d V \\
& \quad-\frac{1}{4 n} \int_{M} \mathscr{L} \mathscr{L}\left(a f+\frac{n-1}{K} a^{\prime} \Delta f+b g+\frac{n-1}{K} b^{\prime} \Delta g\right) d V \\
& \quad+\frac{(n-1)(n+2)}{4 K} \int_{M} \rho_{i} \rho^{i}\left(a^{\prime} f+b^{\prime} g\right) d V,
\end{aligned}
$$

$a, a^{\prime}, b, b^{\prime}$ being constants. Now we choose these constants so as to have

$$
\alpha_{0}=a, \quad \alpha_{1}=\frac{n-1}{K} a^{\prime}, \quad \beta_{0}=b, \quad \beta_{1}=\frac{n-1}{K} b^{\prime} .
$$

Then from (4.16) we find

$$
\begin{array}{ll}
4 a-(n+6) a^{\prime} \geq 0, & a^{\prime} \geq 0, \\
4 b-(n+6) b^{\prime} \geq 0, & b^{\prime} \geq 0,
\end{array}
$$

and

$$
\begin{aligned}
& 4\left(a-a^{\prime}\right) \geq(n+2) a^{\prime} \geq 0, \\
& 4\left(b-b^{\prime}\right) \geq(n+2) b^{\prime} \geq 0,
\end{aligned}
$$

and consequently

$$
\frac{n-2}{2}\left(a-a^{\prime}\right)+2\left(b-b^{\prime}\right) \geq 0,
$$

the equality sign occurring when and only when $a=a^{\prime}=b=b^{\prime}=0$, that is, $\alpha_{0}=\alpha_{1}=\beta_{0}=\beta_{1}=0$. 
Thus from the assumption and (4.19), we have

$$
\int_{M} G_{j i} \rho^{j} \rho^{i} d V \geq 0
$$

and consequently, by theorem $\mathrm{D}, M$ is isometric to a sphere.

Remark 4.1. If

$$
\mathscr{L}\left(\alpha_{0} f+\alpha_{1} \Delta f+\beta_{0} g+\beta_{1} \Delta g\right)=\text { constant, }
$$

then (4.15) is automatically satisfied. But if $\mathscr{L} h=$ constant for a scalar field $h$ in a compact space, the constant must be zero, because $h$ attains an extreme value at a certain point of the space at which $\mathscr{L} h=v^{i} \nabla_{i} h=0$. The same remark applies to Theorems E, 4.1, 5.1, 6.1, 6.2 and 6.4.

\section{A theorem similar to that of Hsiung}

To obtain Theorem F, Hsiung [2] used the tensor

$$
a Z_{k j i h}+b g_{k h} G_{j i}
$$

but we would like to use here the tensor

$$
W_{k j i h}=a Z_{k j i h}+\frac{b}{n-2}\left(g_{k h} G_{j i}-g_{j h} G_{k i}+G_{k h} g_{j i}-G_{j h} g_{k i}\right)
$$

$a$ and $b$ being constants.

It is easily seen that

$$
W_{k j i h} g^{k h}=(a+b) G_{j i},
$$

and that, when $a+b=0$,

$$
W_{k j i h}=a C_{k j i h},
$$

where

$$
\begin{aligned}
C_{k j i h}= & K_{k j i h}-\frac{1}{n-2}\left(g_{k h} K_{j i}-g_{j h} K_{k i}+K_{k h} g_{j i}-K_{j h} g_{k i}\right) \\
& +\frac{K}{(n-1)(n-2)}\left(g_{k h} g_{j i}-g_{j h} g_{k i}\right)
\end{aligned}
$$

is the covariant Weyl conformal curvature tensor.

In general, we have 


$$
W_{k j i h} W^{k j i h}=a^{2} Z_{k j i h} Z^{k j i h}+\frac{4(2 a+b) b}{n-2} G_{j i} G^{j i}
$$

and for the case $a+b=0$ we have

$$
W_{k j i h} W^{k j i h}=a^{2} C_{k j i h} C^{k j i h} .
$$

Using the tensor $W_{k j i h}$ defined above we can obtain

Theorem 5.1. Suppose that a compact orientable $M$ of dimension $n>2$ with $K=$ const. admits an infinitesimal nonhomothetic conformal transformation $v^{h}$. If

$$
\mathscr{L} \mathscr{L}\left(W_{k j i h} W^{k j i h}\right) \leq 0
$$

or equivalently,

$$
(n-2) a^{2} \mathscr{L} \mathscr{L}\left(Z_{k j i h} Z^{k j i h}\right)+4(2 a+b) b \mathscr{L} \mathscr{L}\left(G_{j i} G^{j i}\right) \leq 0,
$$

$a$ and $b$ being constants such that $a+b \neq 0, M$ is isometric to a sphere.

To prove this theorem, we need the following

Lemma 5.1. For an infinitesimal conformal transformation $v^{h}$ in $M: \mathscr{L}_{g_{j i}}$ $=2 \rho g_{j i}$, we have

$$
\begin{aligned}
\mathscr{L} W_{k j i h}= & 2 a \rho Z_{k j i h}+\frac{2 b \rho}{n-2}\left(g_{k h} G_{j i}-g_{j h} G_{k i}+G_{k h} g_{j i}-G_{j h} g_{k i}\right) \\
& -(a+b)\left(g_{k h} \nabla_{j} \rho_{i}-g_{j h} \nabla_{k} \rho_{i}+\nabla_{k} \rho_{h} g_{j i}-\nabla_{j} \rho_{h} g_{k i}\right) \\
& +\frac{2(a+b)}{n} \Delta \rho\left(g_{k h} g_{j i}-g_{j h} g_{k i}\right) .
\end{aligned}
$$

Proof. This follows from (1.10), (1.11) and

$$
\mathscr{L} Z_{k j i h}=\mathscr{L}\left(Z_{k j i}{ }^{t} g_{t h}\right)=\left(\mathscr{L} Z_{k j i}{ }^{t}\right) g_{t h}+2 \rho Z_{k j i h} .
$$

Lemma 5.2. For an infinitesimal conformal transformation $v^{h}$ in $M: \mathscr{L} g_{j i}$ $=2 \rho g_{j i}$, we have

$$
\left(\mathscr{L} W_{k j i h}\right) w^{k j i h}=2 \rho W_{k j i h} W^{k j i h}-4(a+b)^{2} G_{j i} \nabla^{j} \rho^{i} .
$$

Proof. This follows from (5.5) and (5.9).

Lemma 5.3. For an infinitesimal conformal transformation $v^{h}$ in $M$, we have

$$
\mathscr{L}\left(W_{k j i h} W^{k j i h}\right)=-4 \rho W_{k j i h} W^{k j i h}-8(a+b)^{2} G_{j i} \nabla^{j} \rho^{i}
$$

Proof. This follows from (5.11) and

$$
\mathscr{L}\left(W_{k j i h} W^{k j i h}\right)=2\left(\mathscr{L} W_{k j i h}\right) W^{k j i h}-8 \rho W_{k j i h} W^{k j i h} .
$$


Lemma 5.4. For an infinitesimal conformal transformation $v^{h}$ in $M$ with $K=$ constant, we have

$$
\begin{aligned}
& 8(a+b)^{2} \nabla^{j}\left(G_{j i} \rho \rho^{i}\right) \\
& \quad=8(a+b)^{2} G_{j i} \rho^{j} \rho^{i}-4 \rho^{2} W_{k j i h} W^{k j i h}-\rho \mathscr{L}\left(W_{k j i h} W^{k j i h}\right) .
\end{aligned}
$$

Proof. This follows from $\nabla^{j} G_{j i}=0$ and (5.12).

Lemma 5.5. If a compact orientable $M$ of dimension $n>2$ with $K$ $=$ const. admits an infinitesimal nonhomothetic conformal transformation $v^{h}$, then we have

$$
\begin{aligned}
& 8(a+b)^{2} \int_{M} G_{j i} \rho^{j} \rho^{i} d V \\
& =4 \int_{M} \rho^{2} W_{k j i h} W^{k j i n} d V+\int_{M} \rho \mathscr{L}\left(W_{k j i h} W^{k j i h}\right) d V \\
& =4 \int_{M} \rho^{2} W_{k j i h} W^{k j i n} d V-\frac{1}{n} \int_{M} \mathscr{L} \mathscr{L}\left(W_{k j i h} W^{k j i h}\right) d V .
\end{aligned}
$$

Proof. This follows from (5.13) by integrating both sides over $M$ and using Lemma 2.1.

Proof of the theorem. If $\mathscr{L} \mathscr{L}\left(W_{k j i n} W^{k j i h}\right) \leq 0$, and $a+b \neq 0$, then from (5.14) we have

$$
\int_{M} G_{j i} \rho^{j} \rho^{i} d V \geq 0
$$

Thus by Theorem $\mathrm{D}, M$ is isometric to a sphere.

\section{Characterizations of conformally flat spaces}

Theorem 6.1. If a compact orientable $M$ of dimension $n>3$ admits an infinitesimal conformal transformation $v^{h}: \mathscr{L}_{j i}=2 \rho g_{j i}$ such that $\rho$ does not vanish on any n-dimensional domain and

$$
\mathscr{L} \mathscr{L} h<0, \quad h=C_{k j i h} C^{k j i h},
$$

then $M$ is conformally flat.

Proof. Multiplying (5.12), with $a+b=0$, by $\rho$ and integrating the resulting equation over $M$, we find

$$
0=4 \int_{M} \rho^{2} h d V+\int_{M} \rho \mathscr{L} h d V,
$$

or by Lemma 2.1 , 


$$
0=4 \int_{M} \rho^{2} h d V-\frac{1}{n} \int_{M} \mathscr{L} \mathscr{L} h d V
$$

(6.2) implies

$$
\int_{M} \rho^{2} h d V \leq 0,
$$

from which $\rho^{2} h=0$, or by the assumption of the theorem, $h=0$, that is, $C_{k j i h}=0$, which shows that $M$ is conformally flat.

Remark 6.1. If $\mathscr{L} h=$ constant in a compact space, we have $\mathscr{L} h=0$. On the other hand, if $\mathscr{L} h=0$ in a general Riemannian space, from $\mathscr{L} h+4 \rho h=0$ we find $h=0$, which shows that the space is conformally flat.

Theorem 6.2. Under the same assumptions as in Theorem 6.1, if $K=$ const. and (6.1) is replaced by

$$
\mathscr{L}\left\{\sum_{k=0}^{l} \alpha_{k}\left(-\frac{n-1}{K}\right)^{k} \Delta^{k}(\mathscr{L} h)\right\} \leq 0,
$$

$l$ being a nonnegative integer and $\alpha_{k}$ constants such that $\sum_{k=0}^{l} \alpha_{k}>0$, then $M$ is conformally flat.

Proof. Similarly, as in the proof of Theorem 4.1 we can obtain

$$
\begin{gathered}
0=4 \int_{M}\left(\alpha_{0}+\alpha_{1}+\cdots+\alpha_{l}\right) \rho^{2} h d V \\
\quad+\int_{M} \rho\left\{\alpha_{0} \mathscr{L} h+\alpha_{1}\left(-\frac{n-1}{K}\right) \Delta(\mathscr{L} h)+\cdots\right. \\
\left.\quad+\alpha_{l}\left(-\frac{n-1}{K}\right)^{l} \Delta^{l}(\mathscr{L} h)\right\} d V,
\end{gathered}
$$

or, by virtue of Lemma 2.1 ,

$$
\begin{gathered}
0=4 \int_{M}\left(\alpha_{0}+\alpha_{1}+\cdots+\alpha_{l}\right) \rho^{2} d V \\
-\frac{1}{n} \int_{M} \mathscr{L}\left\{\alpha_{0} \mathscr{L} h+\alpha_{1}\left(-\frac{n-1}{K}\right) \Delta(\mathscr{L} h)+\cdots\right. \\
\left.\quad+\alpha_{l}\left(-\frac{n-1}{K}\right)^{l} \Delta^{l}(\mathscr{L} h)\right\} d V,
\end{gathered}
$$

$\alpha_{0}, \alpha_{1}, \cdots, \alpha_{l}$ being constants such that $\sum_{k=0}^{l} \alpha_{k}>0$. Thus by (6.3), we have $\int_{M} \rho^{2} h d V=0$, from which $h=0$ and consequently $C_{k j i h}=0$. 
Theorem 6.3. Under the same assumptions as in Theorem 6.1 , if $K=$ const. and (6.1) is replaced by

$$
\alpha_{0} h-\alpha_{1} \Delta h=c \text { (constant), }
$$

$\alpha_{0}$ and $\alpha_{1}$ being positive constants such that

$$
\frac{8 K}{n-1} \alpha_{1}>(n-6) \alpha_{0} \geq 0, \quad \text { for } n>6,
$$

then $M$ is conformally flat.

Proof. Similarly, as in the proof of Theorem 4.2 we can obtain

$$
0=\int_{M} \rho^{2}\left[\left\{8 a^{\prime}-(n-6) a\right\} h+(n+2) c\right] d V .
$$

Now, the constant $8 a^{\prime}-(n-6) a$ is positive for $n \leq 6$. Since

$$
8 a^{\prime}-(n-6) a=\frac{8 K}{n-1} \alpha_{1}-(n-6) \alpha_{0},
$$

by (6.6) this constant is also positive for $n>6$.

On the other hand, from (6.5) we have

$$
\alpha \int_{M} h d V=c \int_{M} d V
$$

which shows that $c$ is a nonnegative constant.

Thus from (6.7) we see that $h=0$ and consequently $C_{k j i h}=0$.

Theorem 6.4. Under the same assumption as in Theorem 6.1, if $K=$ const. and (6.1) is replaced by

$$
\mathscr{L} \mathscr{L}\left(\alpha_{0} h+\alpha \Delta h\right) \leq 0,
$$

$\alpha_{0}$ and $\alpha_{1}$ being constants such that

$$
\frac{4(n-1)}{K} \alpha_{0}>(n+6) \alpha_{1} \geq 0,
$$

then $M$ is conformally flat.

Proof. Similarly, as in the proof of Theorem 4.3 we can obtain

$$
\begin{aligned}
0= & \left\{4 a-(n+6) a^{\prime}\right\} \int_{M} \rho^{2} h d V-\frac{1}{n} \int_{M} \mathscr{L} \mathscr{L}\left(a h+\frac{n-1}{K} a^{\prime} \Delta h\right) d V \\
& +\frac{(n-1)(n+2)}{K} \int_{M} \rho_{i} \rho^{i}\left(a^{\prime} h\right) d V,
\end{aligned}
$$


$a$ and $a^{\prime}$ being constants. Now we choose these constants such that

$$
\alpha_{0}=a, \quad \alpha_{1}=\frac{n-1}{K} a^{\prime} .
$$

Then from (6.9) we have

$$
4 a-(n+6) a^{\prime}=4 \alpha_{0}-(n+6) \frac{K}{n-1} \alpha_{1}>0 .
$$

We also have

$$
\mathscr{L}\left(a h+\frac{n-1}{K} a^{\prime} \Delta h\right)=\mathscr{L}\left(\alpha_{0} h+\alpha_{1} \Delta h\right)=\text { constant } .
$$

Thus, from (6.10), we have $h=0$ and consequently $C_{k j i h}=0$.

\section{Bibliography}

[1] C. C. Hsiung, On the group of conformal transformations of a compact Riemannian manifold, Proc. Nat. Acad. Sci. U.S.A. 54 (1965) 1509-1513.

[2] - On the group of conformal transformations of a compact Riemannian manifold. II, Duke Math. J. 34 (1967) 337-341.

[3] A. Lichnerowicz, Sur les transformations conformes d'une variété riemannienne compacte, C. R. Acad. Sci. Paris 259 (1964) 697-700.

[4] M. Obata, Certain conditions for a Riemannian manifold to be isometric with a sphere, J. Math. Soc. Japan, 14 (1962) 333-340.

[5] K. Yano, The theory of Lie derivatives and its applications, North-Holland, Amsterdam, 1957.

[6] - On Riemannian manifolds with constant scalar curvature admitting a conformal transformation group, Proc. Nat. Acad. Sci. U.S.A. 55 (1966) 472-476.

[7] K. Yano, \& M. Obata, Sur le groupe de transformations conformes d'une variété de Riemann dont le scalaire de courbure est constant, C. R. Acad. Sci. Paris 260 (1965) 2698-2700.

TOKYo Institute OF TECHNOLOGY Nigata UNIVERSITY 